\title{
Predictive value of high-sensitive troponin $t$ versus conventional biomarkers for 1-year left ventricular function and infarct size after STEMI
}

\author{
Sebastian J Reinstadler ${ }^{1 *}$, Gert Klug ${ }^{1}$, Hans-Josef Feistritzer ${ }^{1}$, Alexander Tu' ${ }^{1}$ Michael Schocke², \\ Wolfgang-Michael Franz ${ }^{1}$, Bernhard Metzler ${ }^{1}$ \\ From 18th Annual SCMR Scientific Sessions \\ Nice, France. 4-7 February 2015
}

\section{Background}

Data relating high-sensitive troponin $\mathrm{T}$ (hs-cTnT) to long-term myocardial function and damage in patients after STEMI are lacking. We evaluated the use of serial and peak concentrations of hs-cTnT versus creatine kinase (CK), lactate dehydrogenase (LDH) and high sensitive C-reactive protein (hs-CRP) for prediction of myocardial function as well as infarct scar assessed by cardiac magnetic resonance imaging (CMR) one year after first ST-segment elevation myocardial infarction (STEMI).

\section{Methods}

Sixty-six patients receiving primary percutaneous coronary intervention ( $\mathrm{p}-\mathrm{PCI}$ ) for first STEMI were enrolled in this single-centre, observational study. All participants underwent cine and contrast-enhanced CMR within the first week and 12 months after the index event. Serial biomarkers were determined on admission, $6 \mathrm{~h}, 12 \mathrm{~h}, 24 \mathrm{~h}$, and 12 months following p-PCI. Hs-cTnT concentrations were measured by a fourth generation high-sensitive immunoassay (Roche Diagnostics ${ }^{\circledR}$ ). Other biomarkers were assessed using commercially available assays.

\section{Results}

Except for admission values, all single time point and peak hs-cTnT concentrations showed moderate to good correlations with 12-months left ventricular ejection fraction (LVEF) ( $\mathrm{r}=-0.41$ to -0.52 , all $\mathrm{p}<0.01$ ) and infarct size (IS) $(\mathrm{r}=0.50$ to 0.70 , all $\mathrm{p}<0.01$ ). Peak CK (LVEF: $\mathrm{r}=-0.45$ to -0.56 , IS: $r=0.61$ to 0.65 ), peak LDH (LVEF: $r=-0.51$ to

'University Clinic of Internal Medicine III, Cardiology and Angiology, Medical University Innsbruck, Innsbruck, Austria

Full list of author information is available at the end of the article
-0.59, IS: $\mathrm{r}=0.54$ to 0.69 ), and peak hs-CRP (LVEF: $\mathrm{r}=$ -0.32 to -0.36 , IS: $r=0.31$ to 0.36 ) were also significantly related with CMR parameters (all $\mathrm{p}<0.05$ ). In receiveroperator characteristic analysis, peak hs-cTnT (AUC = $0.82(0.71-0.92)$ and $0.89(0.81-0.97)$, respectively) and peak LDH (AUC $=0.83(0.74-0.93)$ and 0.91 (0.84 - 0.99), respectively) displayed the best performance for prediction of reduced LVEF $(<55 \%, \mathrm{n}=29)$ and large infarct size at follow-up ( $>8 \%$ of left ventricular myocardial mass, $\mathrm{n}=$ $36)$. The combination of biomarkers did not significantly improve the predictive power of hs-cTnT alone $(\mathrm{p}>0.05)$.

\section{Conclusions}

In patients with first STEMI, peak concentrations of hscTnT are closely correlated to long-term myocardial function and infarct size. Maximum concentrations of CK, LDH, and hs-CRP were also correlated with 12months LVEF and infarct size, but did not add any significant prognostic value compared to hs-cTnT alone.

\section{Funding}

Austrian Society of Cardiology.

\begin{abstract}
Authors' details
'University Clinic of Internal Medicine III, Cardiology and Angiology, Medical University Innsbruck, Innsbruck, Austria. ${ }^{2}$ Departement of Radiology I, Medical University of Innsbruck, Innsbruck, Austria.
\end{abstract}

Published: 3 February 2015

\section{doi:10.1186/1532-429X-17-S1-P178}

Cite this article as: Reinstadler et al:: Predictive value of high-sensitive troponin $\mathrm{t}$ versus conventional biomarkers for 1-year left ventricular function and infarct size after STEMI. Journal of Cardiovascular Magnetic Resonance 2015 17(Suppl 1):P178. 